\title{
Relação entre fatores de personalidade e estratégias de coping em adolescentes
}

\author{
Simon Santana Diniz. - Pontifícia Universidade Católica de Goiás, Goiás, Brasil \\ Daniela S. Zanini - Pontifícia Universidade Católica de Goiás, Goiás, Brasil
}

\begin{abstract}
Resumo
O objetivo deste estudo é investigar a relação entre fatores de personalidade e estratégias de coping em adolescentes. Participaram desta pesquisa 102 jovens de uma escola municipal de Goiânia com idade entre 11 e 15 anos, utilizando o Coping Response Inventory e a Bateria Fatorial de Personalidade. Os dados obtidos demonstraram que meninas utilizam mais a análise lógica para resolver seus problemas e os meninos apresentam maior pontuação em neuroticismo; que os adolescentes mais jovens utilizam mais coping de evitação e os mais velhos, o coping de aproximação; e que tanto a apreciação do problema como os traços de personalidade relacionam-se significativamente com o uso de estratégias de coping. Os resultados são discutidos de acordo com as teorias de coping.

Palavras-chave: Estratégias de enfrentamento, Personalidade, Cinco grandes fatores, Adolescência.
\end{abstract}

\section{Relationship between personality factors and coping strategies in adolescents}

\begin{abstract}
The aim of this study is to investigate the relationship between personality factors and coping strategies in adolescents. 102 students from a municipal school in Goiânia aged between 11 and 15 years old was assessed using Coping Response Inventory and the "Bateria Fatorial de Personalidade". Data showed that girls use more logical analysis to solve their problems and that boys had higher scores on neuroticism; that younger adolescents use more avoidance coping responses and older ones use more approach coping responses; and that both coping appraisal and personality traits are significantly related to the use of coping strategies. The results are discussed according to coping theories.

Keywords: Coping, Personality, Big-five, Adolescence.
\end{abstract}

A maneira como os adolescentes enfrentam seus problemas, referida na literatura em psicologia como estratégias de enfrentamento a problemas ou coping, vem sendo estudada nas mais diversas culturas, demonstrando que existe ampla variedade de comportamentos atenuadores das vivências de estresse ou utilizados no confronto a situações adversas. Além disso, o reconhecimento de que os períodos da infância e da adolescência são também permeados por questões psicossociais que predispõem os jovens ao desenvolvimento de psicopatologias (Marcelli, 2007) impulsionou diversas pesquisas sobre o tema nesta etapa do desenvolvimento. Algumas delas buscam conhecer quais fatores poderiam exercer influência na escolha das maneiras como os indivíduos enfrentam seus problemas.

Os estudos sobre coping tiveram sua origem nas pesquisas sobre estresse. Tornaram-se mais sistematizados a partir de 1984, quando Richard Lazarus e Susan Folkman elaboraram uma das definições mais aceitas e utilizadas até os dias de

\footnotetext{
${ }^{1}$ Endereço para correspondência:

Departamento de Psicologia, Universidade Católica de Goiás, Avenida Universitária 1069, Setor Universitário - Caixa Postal 86 74605-010 - Goiânia-GO

E-mail: dazanini@yahoo.com
}

hoje. Segundo esses autores, coping é definido como "mudanças constantes em esforços cognitivos ou comportamentais para enfrentar demandas específicas externas e/ou internas que são apreciadas pelo indivíduo como sobrecarregando ou excedendo seus próprios recursos" (Lazarus \& Folkman, 1984, p. 141). Neste sentido, o processo de coping envolve quatro conceitos básicos: $1^{\circ}$ ) o coping é um processo que ocorre entre indivíduo e ambiente, $2^{\circ}$ ) a função do coping é administrar a situação estressora, $3^{\circ}$ ) o processo de coping pressupõe que o indivíduo percebeu, interpretou e representou cognitivamente a situação avaliada como estressante, e $4^{\circ}$ ) o processo de coping constitui uma mobilização de esforços cognitivos e comportamentais para administrar o evento adverso, ou seja, para que ocorra a elaboração das estratégias de enfrentamento, o indivíduo deve perceber o evento estressante, avaliar suas opções e, por fim, lançar mão daquela estratégia avaliada como mais apropriada para o momento (Compas, Connor-Smith, Saltzman, Thomsen \& Wadsworth, 2001).

A teoria de Lazarus e Folkman (1984) propõe que um dado evento pode ser apreciado como irrelevante, benigno ou estressante. No caso de se considerar este último, a situação pode ser 
percebida pelo indivíduo como uma ameaça, um prejuízo ou um desafio. Este processo é chamado de avaliação primária. Com base nela, o indivíduo tende a buscar formas de administrar o evento, considerando o que pode ser feito sobre a situação, qual é o custo emocional envolvido e quais são os resultados esperados desse processo. Esta é chamada avaliação secundária (Antoniazzi, Dell'Aglio \& Bandeira, 1998). O comportamento e as atitudes procedentes dessa reflexão são as estratégias de coping que, segundo Lazarus e Folkman (1984), podem ser focadas na emoção (quando os esforços do sujeito são dirigidos a minimizar as consequências emocionais resultantes da experiência com o evento estressante) ou no problema (quando os esforços são dirigidos para o problema em si, na tentativa de manejar ou reduzir o evento estressor).

Estudos têm demonstrado que a forma pela qual crianças e adolescentes lidam com situações adversas é de extrema importância para a manutenção de sua saúde física e psíquica (Raimundo \& Pinto, 2006), assim como para a sua adaptação social e relações interpessoais (Dell'Aglio, 2003; Dell'Aglio \& Hutz, 2002a; Dell'Aglio \& Hutz, 2002b). Apesar disso, Compas e cols. (2001) afirmam que os estudos sobre coping em crianças são escassos, pois há uma tendência a acreditar que crianças não sofrem tantas tensões como os adultos.

A compreensão do coping em crianças e adolescentes envolve diversas facetas. Pesquisas têm demonstrado que fatores como sexo ou gênero, idade e personalidade exercem grande influência na escolha das estratégias. Eschenbeck, Kohlmann e Lohaus (2007) demonstram que meninos e meninas utilizam formas distintas de coping para administrar as mesmas situações de estresse. Os primeiros geralmente utilizam mais estratégias focadas no problema; em contrapartida, as meninas utilizam mais estratégias focadas na emoção.

Kochenderfer-Ladd e Skinner (2002) defendem que estratégias de coping em crianças podem ter diferentes repercussões, dependendo do contexto social e de aspectos pessoais dos indivíduos. Nesse sentido, podemos dizer que uma forma de enfrentamento ao problema pode ser bem aceita para uma menina, como manifestar suas emoções chorando, por exemplo, e ao mesmo tempo ser encarada como sinal de fraqueza para um menino. Em relação a este aspecto Câmara e Carlotto (2007) discutem o papel mediador do gênero não só em relação à escolha das estratégias de coping, mas também no que tange à influência das respostas de coping sobre o bem-estar psicológico do adolescente. Esses autores postulam que adolescentes do gênero masculino e feminino, ademais de utilizarem diferentes estratégias de enfrentamento aos problemas, também sofrem, diferentemente, as influências do uso dessas estratégias no bem-estar psicológico.

Com relação à idade, Boo e Wicherts (2007) mencionam que crianças mais velhas tendem a buscar soluções de problemas mais do que evitam pensar sobre eles. Esses dados corroboram os encontrados por Dell'Aglio e Hutz (2002b) em população adolescente brasileira. Esses autores encontraram que crianças e adolescentes entre 11 e 15 anos tendem a usar mais estratégias de enfrentamento direto para lidarem com seus problemas, mas ressaltam o papel situacional das respostas de coping e a influência do tipo de evento estressante vivenciado na determinação da estratégia de coping utilizada. Também em população adolescente brasileira, Lisboa e cols. (2002) encontraram que os adolescentes mais novos usam significativamente mais estratégias de coping focadas na emoção e justificaram o uso aumentado de tais estratégias pelo fato desse grupo não possuir, ainda, recursos cognitivos eficientes para agir sobre os eventos estressores.

Com relação à influência da personalidade na escolha das estratégias de coping, parece que algumas características do desenvolvimento cognitivo e social tendem a afetar não só o que as crianças e adolescentes experimentam como evento estressante, mas também a forma como lidam com essas situações (Compas \& cols., 2001; Dell'Aglio \& Hutz, 2002a; 2002b). Entre estas características podemos citar o lócus de controle ou a atribuição de causalidade (Lazarus \& Folkman, 1984). Há evidências de que o lócus de controle interno, ou seja, a avaliação de que o problema foi causado pelo próprio indivíduo, está relacionada às estratégias de coping de aproximação (Moos, 1993) ou focadas no problema (Aldwin, 1994; Lazarus \& Folkman, 1984), enquanto a apreciação de que o problema foi causado por outra pessoa relaciona-se positivamente com estratégias de coping focadas na emoção ou do tipo evitativa (Lazarus \& Folkman, 1984; Moos, 1993).

Por outro lado, nos últimos anos viu-se crescer o interesse nos estudos sobre a influência da personalidade na saúde dos indivíduos. Grande parte deste interesse se viu renovado pelo desenvolvimento do modelo do Big Five ou Cinco Grandes Fatores (McCrae \& Costa, 1986). O 
modelo do Big Five deriva da Teoria de Traço e, segundo Hutz e cols. (1998), constitui um avanço conceitual e empírico no campo de estudo da personalidade. Esse modelo descreve cinco dimensões humanas básicas de forma consistente e replicável. São elas: neuroticismo (ou instabilidade emocional), extroversão (ou intensidade das interações interpessoais), Socialização (ou quantidade da orientação interpessoal do indivíduo), realização (ou grau de organização, persistência e motivação do indivíduo) e abertura (ou atividade proativa) (Nunes \& cols., no prelo).

Estudos recentes discutem ao menos duas formas de como essa influência da personalidade sobre a saúde pode ocorrer. Uma, por meio da influência direta, ou seja, indivíduos com determinados traços de personalidade tendem a desenvolver determinadas doenças (Nunes, 2005); e outra por meio do efeito mediador ou moderador do coping, isto é, indivíduos com determinadas características ou traços de personalidade tendem a enfrentar os problemas de sua vida de determinada maneira e esta forma de enfrentamento específica repercute em sua saúde (Zanini, 2003). Nesse sentido, estudos com população adulta apontam para a existência de uma relação entre neuroticismo e os processos de estresse e coping. Por exemplo, Aldwin (1994) demonstrou que indivíduos com alta pontuação em neuroticismo tendem a perceber mais eventos como estressores e McCrae e Costa (1986) encontraram correlação entre neuroticismo e formas de enfretamento consideradas passivas, evitativas e ineficazes.

Dados similares foram encontrados por Endler e Parker (1990), que informam uma relação entre neuroticismo e um aumento no uso de estratégias centradas na emoção em ambos sexos e uma diminuição do uso de coping centrado no problema nas mulheres. Em outros estudos, Carver, Scheier e Weintraub (1989) e Watson e Hubbard (1996) encontraram correlações positivas entre neuroticismo e ventilação de emoções, desvinculação comportamental e mental e negação, e correlações negativas entre esse traço de personalidade e estratégias do tipo aceitação, reinterpretação e, em um nível menor, planejamento. Esses autores também assinalam a existência de importantes correlações positivas entre consciência e as estratégias de planejamento e coping ativo, assim como entre extroversão e reinterpretação positiva, busca de apoio emocional e busca de apoio instrumental.
Em população adolescente ainda são escassos os estudos que avaliam a influência da personalidade sobre o uso das estratégias de coping. Contudo, os poucos estudos existentes apresentam dados semelhantes àqueles encontrados em população adulta. Por exemplo, Gomez, Bounds, Holmberg, Fullarton e Gomez (1999a) encontraram uma correlação positiva entre neuroticismo e estilo de coping evitativo. Esses mesmos autores estudaram a influência do neuroticismo e extroversão como preditores dos estilos de coping, encontrando correlações elevadas entre neuroticismo e o estilo de coping evitativo, sobretudo para as mulheres. Também encontraram correlações positivas entre a extroversão e o uso de estratégias de coping tanto cognitivas como comportamentais, porém desta vez, em níveis semelhantes para meninos e meninas (Gomez, Holmberg, Bounds, Fullarton \& Gomez, 1999b). Porém, cabe ressaltar que os níveis de explicação encontrados para personalidade sobre as estratégias de coping são muito inferiores aos descritos na bibliografia de adultos.

Com base no exposto, o presente estudo propõe-se investigar a relação entre fatores de personalidade e estratégias de coping em adolescentes, avaliados por meio dos traços de personalidade e da apreciação do problema, além das variáveis sexo e idade.

\section{Método}

\section{Participantes}

Participaram deste estudo 102 alunos dos ensinos fundamental e médio de uma escola municipal de tempo integral da cidade de Goiânia, com idade entre 11 e 15 anos $(M=12,80$; $\mathrm{DP}=0,98)$. Os alunos pertencem a camadas de nível socioeconômico baixo, médio-baixo e médio (que foram definidos com base na faixa salarial dos pais, segundo dados da própria escola), sendo de maioria étnica branca (autoinforme) e categorizados como $59 \%$ do sexo masculino e $41 \%$ do sexo feminino.

\section{Instrumentos}

Foram utilizados para este estudo o Coping Response Inventory-Y (CRI-Y) e a Bateria Fatorial de Personalidade (BFP) - versão preliminar. O CRI $\mathrm{Y}$ de Moos (1993) visa medir os esforços cognitivos e comportamentais dos indivíduos no enfrentamento a problemas e divide-se em três partes: a) descrição da situação mais estressante ou difícil vivenciada nos últimos 12 meses pelo 
adolescente; b) dez perguntas de apreciação do problema relativas à experiência prévia com o problema, prejuízos causados por este, lócus de controle, se o problema já está resolvido, entre outras; c) quarenta e oito perguntas relativas à descrição de comportamentos que caracterizam as formas de enfrentamento de problemas utilizadas pelas adolescentes. Essas perguntas são respondidas por meio de uma escala Likert de quatro pontos $\mathrm{e}$ as respostas se agrupam, posteriormente, pela soma direta dos itens, em oito escalas diretas de estratégias de coping: análise lógica, reavaliação positiva, busca de orientação e apoio, solução de problemas, avaliação cognitiva, aceitação ou resignação, busca de gratificação alternativa e descarga emocional. $\mathrm{O}$ primeiro grupo de quatro escalas mede o coping de aproximação ao problema ou enfrentamento direto (análise lógica, reavaliação positiva, busca de orientação e apoio e resolução de problemas); o segundo grupo de quatro escalas (evitação cognitiva, aceitação-resignação, busca de gratificação alternativa e descarga emocional) mede o coping de evitação ao problema ou enfrentamento indireto. As duas primeiras escalas em cada grupo medem estratégias de coping cognitivas; a terceira e quarta escalas em cada grupo medem estratégias de coping comportamental (Moos, 1993).

O CRI-Y (Moos, 1993) foi traduzido e validado para o português. A versão resultante em português foi submetida a backtranslation e conta com a aprovação dos autores e editores do instrumento. $\mathrm{O}$ instrumento original apresenta uma qualidade psicométrica satisfatória e concordante com a versão original (do instrumento), com alfas de Cronbach variando entre 0,45 e 0,79 para meninos e 0,50 e 0,79 para meninas.

A Bateria Fatorial de Personalidade (BFP) de Nunes e cols. (no prelo) está sendo desenvolvida pelo Laboratório de Psicologia da UFRGS (Universidade Federal do Rio Grande do Sul), encontrando-se em fase de validação. Consta de um questionário com 167 perguntas que devem ser respondidas levando-se em conta o quanto o sujeito considera que uma resposta é adequada a ele ou não em uma escala Likert de 7 pontos. Foram realizadas análises fatoriais exploratórias para a extração dos fatores e a identificação dos itens mais representativos para os mesmos e para as suas facetas. Para a seleção dos itens, foi considerada a sua carga fatorial, bem como a sua interpretabilidade no fator onde foi alocado. Itens com cargas fatoriais inferiores a 0,30 em todos os fatores ou que apresentavam cargas superiores a esse valor em mais de um fator foram eliminados. O fator neuroticismo apresenta alfa de 0,89; a extroversão apresentou alfa de 0,84 ; a socialização alfa de 0,84 ; a realização alfa de 0,82 e a abertura para experiências alfa de 0,71 (Nunes \& cols., no prelo).

\section{Procedimento}

Foi realizado um contato prévio com a Secretaria Municipal de Educação, solicitando autorização para a realização do estudo em uma das escolas credenciadas. Na escola indicada pela Secretaria, o projeto foi exposto à diretoria e ao corpo docente, com o objetivo de auxiliarem na explicação dos procedimentos e objetivos aos alunos e pais que eventualmente procurassem a escola. Os alunos foram convidados a participar do estudo, tendo em mãos uma carta-convite (que explicava a intenção do projeto, os procedimentos e a necessidade da colaboração dos mesmos), o Termo de Consentimento Livre e Esclarecido e um documento informativo sobre a questão do anonimato resguardado aos participantes, assim como a possibilidade de retirada do consentimento a qualquer momento do processo sem nenhum dano para os mesmos. Também foi oferecida a possibilidade de maiores explicações acerca do estudo em andamento, a qualquer momento, por meio do contato disponibilizado com o pesquisador responsável.

Para este estudo foram realizados dois encontros, que perfaziam um total de 120 minutos aproximadamente, com os grupos participantes. No primeiro encontro ocorreu a aplicação da Bateria Fatorial de Personalidade - BFP. No segundo encontro realizou-se a aplicação do Coping Responses Inventory-Youth Form - CRI-Y.

\section{Resultados}

Para avaliar a existência de diferenças nos traços de personalidade e respostas de coping segundo o sexo foi realizada uma análise de comparação de médias por meio da técnica $T$ de Student. A Tabela 1 apresenta a comparação das médias obtidas ao analisar cada um dos traços de personalidade referenciados no modelo dos Cinco Grandes Fatores e as estratégias de coping de acordo com a variável sexo. 
Tabela 1 - Comparação de médias entre fatores de personalidade e respostas de coping por sexo

\begin{tabular}{lcccc}
\hline Variáveis & \multicolumn{2}{c}{ Média (DP) } & Teste T \\
\cline { 2 - 5 } & $\begin{array}{c}\text { Masculino } \\
\text { (N=61) }\end{array}$ & $\begin{array}{c}\text { Feminino } \\
(\mathrm{N}=41)\end{array}$ & $\mathrm{F}$ & $p<$ \\
\hline Personalidade & & & & \\
$\quad$ Neuroticismo & $78,25(19,61)$ & $73,69(27,30)$ & 7,81 & 0,01 \\
Extroversão & $71,36(17,75)$ & $78,35(15,27)$ & 0,45 & 0,50 \\
Socialização & $30,94(25,21)$ & $23,10(21,97)$ & 1,53 & 0,22 \\
Realização & $34,96(24,91)$ & $36,84(28,87)$ & 1,83 & 0,18 \\
Abertura & $37,69(25,51)$ & $43,47(28,50)$ & 1,70 & 0,19 \\
\hline Estratégias de coping & & & & \\
Análise lógica & $11,72(4,89)$ & $12,45(6,35)$ & 7,61 & 0,01 \\
Reavaliação positiva & $12,93(5,00)$ & $13,97(5,96)$ & 2,52 & 0,12 \\
Busca de apoio & $10,47(6,01)$ & $11,05(6,15)$ & 0,35 & 0,56 \\
Resolução de problemas & $12,93(5,18)$ & $13,32(5,75)$ & 1,11 & 0,29 \\
Evitação cognitiva & $12,87(4,82)$ & $14,42(5,16)$ & 0,30 & 0,58 \\
Aceitação/resignação & $11,07(4,85)$ & $12,19(5,48)$ & 0,48 & 0,49 \\
Busca de gratificação alternativa & $12,78(4,81)$ & $14,92(5,27)$ & 0,13 & 0,72 \\
Descarga emocional & $10,29(5,72)$ & $11,45(5,27)$ & 1,10 & 0,28 \\
\hline
\end{tabular}

Conforme os dados demonstram, não existem diferenças significativas por sexo em quatro dos cinco fatores de personalidade. A única excessão foi para o traço de personalidade neuroticismo que, neste estudo, apresentou-se mais alto entre os meninos quando comparados com as meninas $(F=7,81$ e $p=0,01)$. Da mesma maneira foi encontrada diferença significativa entre coping e sexo apenas para a estratégia de análise lógica - sendo as mulheres as que utilizaram significativamente mais este tipo de estratégia que os homens $(\mathrm{F}=7,61 \mathrm{e} \mathrm{p}=0,01)$.

Para avaliar a diferença nos traços de personalidade e uso de coping segundo a idade foi realizado um teste de ANOVA. A Tabela 2 apresenta os dados dessa análise.

Tabela 2 - Comparação de média entre fatores de personalidade e estratégias de coping por grupos de idade

\begin{tabular}{|c|c|c|c|c|c|}
\hline \multirow[t]{2}{*}{ Variáveis } & \multicolumn{3}{|c|}{ Média(DP) } & \multicolumn{2}{|c|}{ ANOVA } \\
\hline & $\begin{array}{c}11-12 \\
(\mathrm{~N}=39)\end{array}$ & $\begin{array}{c}13 \\
(\mathrm{~N}=39)\end{array}$ & $\begin{array}{c}14-15 \\
(\mathrm{~N}=24)\end{array}$ & $\mathrm{F}$ & $p<$ \\
\hline \multicolumn{6}{|l|}{ Personalidade } \\
\hline Neuroticismo & $80,21(20,56)$ & $72,71(25,84)$ & $78,21(20,00)$ & 1,12 & 0,329 \\
\hline Extroversão & $71,85(14,39)$ & $75,48(19,80)$ & $74,56(16,49)$ & 0,42 & 0,655 \\
\hline Socialização & $19,76(17,91) a$ & $35,78(28,10) b$ & $29,48(23,39)$ & 4,30 & 0,016 \\
\hline Realização & $34,19(25,37)$ & $34,19(24,15)$ & $43,77(31,31)$ & 1,30 & 0,278 \\
\hline Abertura & $31,25(21,66) \mathrm{a}$ & $41,40(27,70)$ & $50,99(28,59) \mathrm{c}$ & 4,40 & 0,015 \\
\hline \multicolumn{6}{|l|}{ Estratégias de coping } \\
\hline Análise lógica & $10,67(5,19)$ & $12,74(5,92)$ & $12,61(5,22)$ & 1,42 & 0,248 \\
\hline Reavaliação positiva & $13,45(5,40)$ & $13,11(5,72)$ & $13,17(4,89)$ & 0,04 & 0,963 \\
\hline Busca de apoio & $9,70(5,71)$ & $10,09(6,31)$ & $12,63(5,68)$ & 1,91 & 0,154 \\
\hline Res. de problemas & $12,58(5,47)$ & $12,53(5,47)$ & $14,63(5,21)$ & 1,30 & 0,278 \\
\hline Evitação cognitiva & $14,00(4,83)$ & $12,97(5,22)$ & $13,54(5,11)$ & 0,35 & 0,707 \\
\hline Aceitação/resignação & $10,38(5,00)$ & $11,49(5,03)$ & $12,92(5,27)$ & 1,71 & 0,186 \\
\hline Busca grat. alternativa & $15,39(4,60) \mathrm{a}$ & $11,49(5,07) \mathrm{b}$ & $14,21(4,80)$ & 5,80 & 0,004 \\
\hline Descarga emocional & $11,06(5,99)$ & $10,29(5,57)$ & $10,83(5,04)$ & 0,17 & 0,843 \\
\hline
\end{tabular}

Nota: as diferenças significativas entre médias estão representadas pelos subíndices.

A Tabela 2 indica diferença significativa entre idade e os fatores socialização ( $F=4,297$, $\mathrm{p}=0,016)$ e abertura $(\mathrm{F}=4,397, \mathrm{p}=0,015)$. A análise demonstra que o grupo de adolescentes de 11-12 
anos apresenta uma pontuação significativamente menor em socialização em relação ao grupo de 13 anos, bem como em relação à abertura, quando comparados com o grupo de 14-15 anos.

A Tabela 2 mostra também que, dentre as estratégias de coping, os adolescentes menores (de 11 e 12 anos) utilizaram significativamente mais a busca de gratificação alternativa como forma de resolver seus problemas quando comparados ao grupo de 13 anos. Com relação às demais estratégias de enfrentamento a problemas não foram encontradas diferenças significativas por grupos de idade.
Para avaliar a relação entre traços de personalidade, a apreciação do problema e estratégias de coping foi realizado um estudo correlacional mediante a técnica de Pearson entre fatores de personalidade, as perguntas de apreciação do problema (Pensou como um desafio? Foi causado por você? Foi causado por outra pessoa? Aconteceu algo de bom? Está tudo bem com você?) e as estratégias de coping. A Tabela 3 apresenta os dados das correlações com níveis de significação inferior a 0.05 .

Tabela 3 - Correlação entre apreciação do problema, perfil psicológico e respostas de coping

\section{Coping}

Variáveis

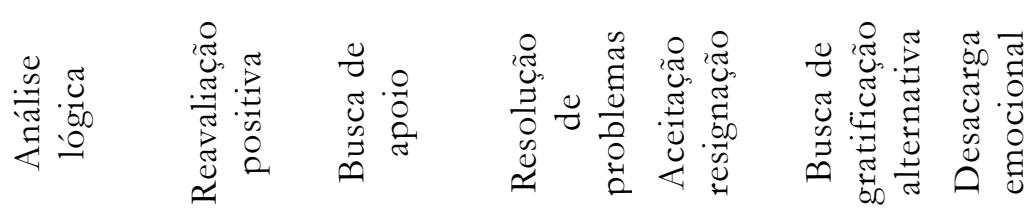

\section{Percepção do problema}

Pensou como um desafio?

Foi causado por você?

Foi causado por outra pessoa?

Aconteceu algo de bom?

Está tudo bem com você?

\section{Personalidade}

Neuroticismo

$0,256 *$

Extroversão

$0,247 * *$

Realização

Abertura

$$
0,349 * *
$$

$0,307 * *$

$0,258 * \quad 0,349 * *$

$0,250^{*}$

$0,230^{*}$

\section{$0,254 * 0,360 * *$}

ota: São apresentadas apenas as variáveis para as quais foram encontradas correlações significativas.

* Significativo no nível de 0,05** Significativo no nível de 0,01

A apreciação do problema correlacionou significativamente com a escolha das seguintes estratégias: aqueles que pensaram o problema como desafio utilizaram mais análise lógica $(\mathrm{r}=0,349 ; \mathrm{p}<0,01)$; busca de apoio $(\mathrm{r}=0,223$; $\mathrm{p}<0,05)$; resolução de problemas $(\mathrm{r}=0,234$; $\mathrm{p}<0,05)$ e aceitação/resignação $(\mathrm{r}=0,215 ; \mathrm{p}<0,05)$. Aqueles que entenderam o problema causado por si mesmos utilizaram mais busca de apoio $(\mathrm{r}=0,216 ; \mathrm{p}<0,05)$ e resolução de problemas $(\mathrm{r}=0,231 ; \mathrm{p}<0,05)$. Os jovens que acreditaram que o problema foi causado por outra pessoa utilizaram mais reavaliação positiva $(r=0,258$; $\mathrm{p}<0,05)$; busca de apoio $(\mathrm{r}=0,349 ; \mathrm{p}<0,01)$; busca de gratificação alternativa $(\mathrm{r}=0,254 ; \mathrm{p}<0,05)$ e descarga emocional $(\mathrm{r}=0,360 ; \mathrm{p}<0,01)$. Os que acreditaram que aconteceu algo bom ao enfrentar a situação utilizaram mais análise lógica $(r=0,307$; $\mathrm{p}<0,01)$ e resolução de problemas $(\mathrm{r}=0,250$; $\mathrm{p}<0,05)$. Por fim, os jovens que consideram que o problema foi resolvido e que está tudo bem utilizaram mais a resolução de problemas $(r=0,230 ; p<0,05)$.

A personalidade correlacionou significativamente na escolha das estratégias: análise lógica (com r=0,256 e $\mathrm{p}=0,16, \mathrm{r}=0,247 \mathrm{e}$ 
$\mathrm{p}=0,26$ e $\mathrm{r}=0,306$ e $\mathrm{p}=0,004$ para neuroticismo, extroversão e abertura, respectivamente), resolução de problemas (com $r=0,214$ e $p=0,49$ e $\mathrm{r}=0,212$ e $\mathrm{p}=0,44$ para realização e abertura, respectivamente) e descarga emocional (com $\mathrm{r}=0,429$ e $\mathrm{p}=0,000$ e $\mathrm{r}=0,280$ e $\mathrm{p}=0,007$ para neuroticismo e abertura, respectivamente).

\section{Discussão}

O objetivo geral deste estudo foi investigar a relação entre fatores de personalidade e estratégias de coping em adolescentes. A avaliação dos traços de personalidade indicou que os adolescentes mais jovens apresentam maior pontuação nos traços socialização e abertura que os demais. Esta diferença pode ser explicada por diferenças no perfil psicológico em relação à idade ou pelas características da amostra acessada. Para o primeiro caso, Marcelli (2007) cita que o desenvolvimento alcançado durante a adolescência sofre influências pessoais, familiares e sociais. Os trabalhos de Compas (1987), Dell'Aglio (2003) e Dell'Aglio e Hutz (2002a; 2002b) também apontam para a influência que o amadurecimento pessoal tem no desenvolvimento de novas condutas durante esse período. No presente estudo tais dados foram confirmados na avaliação dos jovens de 14-15 anos, que apresentaram maior pontuação no traço de abertura que todos os demais $(F=4,40$ e $p=0,015)$, assim como os jovens de 13 anos apresentaram uma pontuação significativamente mais alta que os mais jovens (11-12 anos) no traço socialização $(\mathrm{F}=4,30$ e $\mathrm{p}=0,016)$. Além disso, os jovens de 11-12 anos utilizaram significativamente mais busca de gratificação alternativa que os demais, fato que pode ser justificado pelo menor nível de desenvolvimento psicológico e emocional dos mesmos.

Com relação à variável sexo, neste estudo, contrariamente ao exposto pela literatura em geral, foram os meninos que obtiveram pontuações mais altas no traço neuroticismo quando comparados com as meninas $(\mathrm{F}=7,81$ e $\mathrm{p}=0,01)$ (Hutz \& cols., 1998; Nunes \& col.s, no prelo). Este fato merece especial atenção em estudos futuros, sobretudo a fim de que se esclareça se a maior pontuação encontrada para os meninos neste estudo se deveu ao acaso ou às características específicas desta amostra, ou se, por outro lado, se devem às diferenças culturais dos adolescentes brasileiros.

Além disso, a análise do uso de estratégias de coping revelou diferença significativa apenas para a estratégia de coping análise lógica, sendo as meninas aquelas que mais utilizam esta forma de enfrentamento a problemas $(F=7,61$ e $p=0,01)$. Estes dados corroboram com os descritos por Zanini (2003) em população espanhola. Por outro lado, no geral, as meninas demonstram utilizar mais as estratégias de coping que os meninos, embora em níveis não significativos estatisticamente. Eschenbeck e cols. (2007), Kochenderfer-Ladd e Skinner (2002) citam também esses mesmos resultados e sugerem que esse fato talvez seja decorrente da forma de socialização diferenciada entre os gêneros. Meninos são incentivados a ser mais independentes do que meninas, buscando menos ajuda na resolução de seus problemas.

Aldwin (1994) demonstra que o adolescente reflete sobre seus problemas e sobre como solucioná-los. O presente estudo, em consonância com essa autora, revelou também que a avaliação que o jovem faz a respeito dos eventos adversos influencia na escolha de estratégias de enfrentamento. A análise estatística da apreciação do problema, embasada nas questões propostas no CRI-Y (Moos, 1993), demonstrou correlação positiva com sete das oito estratégias de coping descritas. Dessas correlações, destacam-se: a) avaliar o problema como um desafio (foram significativas a análise lógica, busca de apoio, resolução de problemas e aceitação/resignação) e b) se o problema foi causado por outra pessoa (foram significativas a reavaliação positiva, busca de apoio, busca por gratificação alternativa e descarga emocional). As demais perguntas correlacionaram com número menor de estratégias.

A análise estatística demonstrou que quando se avalia se o evento é entendido como provocado por outra pessoa (lócus de controle externo), o adolescente utiliza significativamente mais a reavaliação positiva, busca de gratificação alternativa e descarga emocional. Segundo Zanini (2003), a utilização de estratégias de evitação ou aquelas com foco na emoção pode ser um indicativo de sofrimento psicológico, uma vez que o jovem não dispôs de recursos eficientes para a resolução da situação-problema. Em contrapartida, estratégias como a reavaliação positiva demonstram o movimento em direção à administração do evento estressante.

A busca de apoio foi significativamente utilizada nas duas situações (lócus de controle interno e externo). Lazarus e Folkman (1984) explicam que a percepção do lócus de controle 
está muito relacionada às crenças individuais. Com base nessa relação, infere-se que o adolescente busca ajuda quando percebe que a situação extrapola seus recursos pessoais e começa a sentirse improdutivo na gerência do evento.

Os dados encontrados demonstraram, ainda, que a personalidade dos jovens estudados exerce influência relativa na eleição de estratégias de coping. Dentre as oito respostas avaliadas, os traços de personalidade indicaram correlação positiva com análise lógica, resolução de problemas e descarga emocional, sendo as primeiras de aproximação e apenas a descarga emocional compondo o repertório de estratégias de evitação. Infere-se, a partir dessas informações, que os traços de personalidade da amostra pesquisada influenciam positivamente na busca por solução do evento estressante. Este fato é de grande interesse para intervenções clínicas voltadas para o desenvolvimento de estratégias de coping. Explorar esses dados pode favorecer o estabelecimento de uma comunicação eficiente com o jovem, auxiliando-o a perceber quais são os recursos que dispõe, aqueles que são mais adequados à situação presente e como fazer o uso mais apropriado destes, alcançando o maior benefício possível.

Um fato que merece destaque refere-se ao perfil abertura. Segundo os dados deste estudo, o traço correlacionou-se positivamente com três das quatro estratégias de aproximação. Hutz e cols. (1998) explicam que este traço "engloba características como flexibilidade de pensamento, fantasia, imaginação, abertura para novas experiências e interesses culturais" (p. 2). Supõese, então, que os fatores abertura, extroversão e realização, em conjunto favoreçam muito o jovem a obter maior suporte $e$ apoio social na administração de problemas, por não manter uma postura rígida ou de esquiva, mas, ao contrário, estando receptivo a novas ideias e informações que o beneficiem. Assim, tais características colocam a seu favor recursos importantes para a administração da situação estressante e consequente manutenção da saúde psicológica. O jovem mais aberto e flexível obtém mais sucesso na gerência de situações adversas, conta com maior apoio por parte da família, tem maior reconhecimento junto ao grupo de pares, além de receber com mais frequência auxílio de terceiros (Zanini, 2003).

Outra correlação que merece atenção é entre o traço realização e a estratégia resolução de problemas. Neste caso, a proposição que se faz para justificá-la é que o indivíduo que possui um tipo de personalidade mais independente, dirigido para a administração dos fatores estressores, tende a buscar a resolução direta de seus problemas.

Concluindo, a literatura específica da área cita mudanças progressivas de comportamento do adolescente, assim como a internalização de estratégias mais elaboradas à medida que o jovem amadurece (Compas \& cols., 2001; Eschenbeck \& cols., 2007). São pouco numerosas ainda as pesquisas envolvendo aspectos da personalidade associados ao comportamento de crianças e adolescentes. Os quadros patológicos que os acometem, ao contrário, possuem amplos estudos que favorecem a intervenção profissional bem sucedida. Entretanto, aspectos mais sutis como os citados, que guardam informações de prevenção muito valiosas a serem utilizadas a seu favor, carecem de maior atenção e exploração por parte dos pesquisadores e clínicos. Tais aspectos são de grande valia para o desenvolvimento de programas preventivos voltados para a população de crianças e adolescentes e que envolvam maior participação da comunidade, da escola e, principalmente, da família. Prevenir o comportamento e os hábitos pouco saudáveis que têm início na juventude resulta em qualidade de vida e saúde na fase adulta.

Conhecer o fato de que o adolescente goiano lida melhor com as situações estressantes quando reflete sobre elas e que sua personalidade tem influência sobre a forma como enfrenta seus problemas e, específicamente, influencia o uso de estratégias de coping diretas, colabora bastante para traçar novas linhas de pesquisa, novas propostas de cuidado e atenção e novas condutas terapêuticas. Este aspecto favorece também uma visão mais criteriosa sobre o jovem e sua forma de expressão.

Os resultados obtidos neste estudo reafirmam a necessidade de ampliar a pesquisa acerca das estratégias de coping na adolescência e sua relação com a personalidade. Contudo, devem ser tomados com precaução, pois se baseiam em um estudo correlacional, de desenho transversal e com uma amostragem por acessibilidade. Para análise da influência da personalidade sobre as estratégias de coping, um estudo longitudinal ou com um desenho experimental e com critério amostral probabilístico seria mais apropriado. Entretanto, dificuldades metodológicas impediram o desenvolvimento desse tipo de estudo neste trabalho. Além disso, estudos futuros devem analisar esses fatores em uma população maior, 
confirmando a solidez das pesquisas preliminares, assim como avaliar a confiabilidade do instrumento de personalidade utilizado e buscar melhores índices de confiabilidade do instrumento de coping a fim de garantir a acuidade e possibilidade de generalização e interpretação dos dados à guisa de influências culturais.

\section{Referências}

Aldwin, C. (1994). Stress, coping and development: an integrative perspective. Nova Yorque: The Guilford Press.

Antoniazzi, A. S., Dell'Aglio, D. D. \& Bandeira, D. R. (1998). A evolução do conceito de coping: uma revisão teórica. Temas de Psicologia, 6(2), 273-294.

Boo, G. M. \& Wicherts, J. M. (2007). Assessing cognitive and behavioral coping strategies in children. Journal Cognitive Therapy and Research. Obtido do World Wide Web: http://www.springerlink.com/content/rm644 $11358433415 /$.

Câmara, S. G. \& Carlotto, M. S. (2007). Coping e gênero em adolescentes. Psicologia em Estudo, Maringá, 12(1), 87-93.

Carver, C. S., Scheier, M. F., \& Weintraub, J. K. (1989). Assessing coping strategies: a theoretically based approach. Journal of Personality and Social Psychology, 56, 267-283.

Compas B. E., Connor-Smith J. K., Saltzman H., Thomsen, A. H. \& Wadsworth, M. E. (2001). Coping with stress during childhood and adolescence: problems, progress and potential in theory and research. Psychological Bulletin, 127(1), 87-127.

Dell'Aglio, D. D. (2003). O processo de coping em crianças e adolescentes: adaptação e desenvolvimento. Temas em Psicologia, 11(1), 3845 .

Dell'Aglio, D. D. \& Hutz, C. S. (2002a). Estratégias de coping e estilo atribucional de crianças em eventos estressantes. Estudos de Psicologia (Natal), 7(1), 5-13.

Dell'Aglio, D. D. \& Hutz, C. S. (2002b). Estratégias de coping de crianças e adolescentes em eventos estressantes com pares e com adultos. Psicologia USP, 13(2), 203-225.

Endler, N. S. \& Parker, J. D. A. (1990). Multidimensional assessment of coping: a critical evaluation. Journal of Personality and Social Psychology, 58, 844-854.

Eschenbeck, H. Kohlmann, C. W. \& Lohaus, A. (2007). Gender differences in coping strategies in children and adolescents. Journal of Individual Differences, 28(1), 18-26.

Gomez, R., Bounds, J., Holmberg, K., Fullarton, C. \& Gomez, A. (1999a). Effects of neuroticism and avoidant coping style on maladjustment during early adolescence. Personality and Individual Differences, 26, 305-319.

Gomez, R., Holmberg, K., Bounds, J., Fullarton, C. \& Gomez, A. (1999b). Neuroticism and extraversion as predictors of coping styles during early adolescence. Personality and Individual Differences, 27, 3-17.

Hutz, C. S., Nunes, C. H., Silveira, A. D., Serra, J., Anton, M. \& Wieczorek, L. S. (1998). O desenvolvimento de marcadores para a avaliação da personalidade no modelo dos cinco grandes fatores. Psicologia: Reflexão e Crítica, 11(2), 395-411.

Kochenderfer-Ladd, B. \& Skinner, K. (2002). Children's coping strategies: moderators of the effects of peer victimization? Developmental Psychology, 38(2), 267-278.

Lazarus, R. \& Folkman, S. (1984). Stress, appraisal and coping. Nova Yorque: Springer.

Lisboa, C., Koller, S. H., Ribas, F. F., Bitencourt, K., Oliveira, L., Porciuncula, L. P. \& De Marchi, R. B. (2002). Estratégias de coping de crianças vítimas e não vítimas de violência doméstica. Psicologia, Reflexão e Crítica, 15(2), 345-362.

Marcelli, D. (2007). Adolescencia e psicopatologia. Porto Alegre: Artmed.

McCrae, R. R. \& Costa, P. T. (1986). Personality, coping, and coping effectiveness in an adult

sample. Journal of Personality, 54, 385-405.

Moos, R. H. (1993). Coping Response Inventory Youth form - professional manual. Odessa: PAR Psychological Assessment Resourses. 
Nunes, C. H. S. S. (2005). Construção, normatização e validação das escalas de socialização e extroversão no modelo dos Cinco Grandes Fatores. Tese de Doutorado, Universidade Federal do Rio Grande do Sul, Porto Alegre.

Nunes, C. H. S. S., Nunes, M. F. O., Primi, R., Muniz, M., Couto, G., Miguel, F. K. \& Hutz, C. S. (no prelo). Bateria Fatorial de Personalidade (BFP) - manual técnico. São Paulo: Casa do Psicólogo.

Raimundo, R. C. P. \& Pinto, M. A. P. M. (2006). Stress e estratégias de coping em crianças e adolescentes em contexto escolar. Aletheia, 24, 9-19.
Watson, D. \& Hubbard, B. (1996). Adaptational style and dispositional structure: coping in the context of the Five-Factor Model. Journal of Personality, 64, 737-774.

Zanini, D. S. (2003). Coping: influencia de la personalidad y repercusiones en la salud mental de los adolescentes. Tese de Doutorado, Universidad de Barcelona, Barcelona.
Recebido em julho de 2009 Reformulado em janeiro de 2010 Aprovado em fevereiro de 2010

Sobre os autores:

Simon Santana Diniz é psicólogo, com mestrado em Psicologia pela Pontifícia Universidade Católica de Goiás, possui pós-graduação em Psicodrama Terapêutico pela PUCGoiás/Sociedade goiana de Psicodrama (2003), graduação em Psicologia (2000) e em Biomedicina (1993) pela PUCGoiás. Atua como psicólogo clínico em clínica privada.

Daniela Sacramento Zanini é psicóloga, com doutorado em Psicologia Clínica e da Saúde e pósdoutorado pela Universidade de Barcelona (Espanha). Atualmente é professora adjunta II no Departamento de Psicologia e Programa de pós-graduação Stricto Sensu - Mestrado em Psicologia, da Pontifícia Universidade Católica de Goiás, orientando em nível de mestrado e doutorado. Atua também como psicóloga no Instituto de Neurologia de Goiânia. 